\title{
A Review of Designing and Implementing an Embedded System Using Client Server and Web Technology for Monitoring and Controlling of Physical Parameters
}

\author{
Siddhartha Baruah \\ Anjana Kakoty Mahanta \\ Kanak Ch Sarma \\ Gauhati University, India
}

\begin{abstract}
Enabling embedded System developed for working in a network environment can provide a lot of opportunities in monitoring and controlling of same from different geographical areas. It may be in a peer to peer type or client server type depending on type of application. Adding web features for such an application, system will be accessible from anywhere from the web. System developed here mainly focus on the issues of possibility of deployment of the system from LAN and Web. Stated study is a microcontroller based embedded system for monitoring and controlling of parameters like temperature and humidity. It can be implemented using simple client server technology as well as web technology. Users belong to the organizational domain can use their own Intranet for monitoring and controlling of parameters designed on simple client server technology whereas users beyond the scope of Intranet can use Internet interface designed using web technology. Both the technologies are having its own advantages as well as disadvantages. This paper explores the technological implications of the system using client server as well as web technology.

Embedded hardware circuit is fabricated in to two separate digital circuits connecting two COM ports of Embedded server. First circuit will monitor temperature and humidity and display same in LCD. Application
\end{abstract}

program executed in computer reads COM port and display the same in monitor and store in database. Second digital circuit will provide necessary hardware control of devices for controlling of temperature and humidity automatically. It is implemented through relay based on the user entered set value of temperature and humidity which is stored in database of computer. Developed system enable its both Intranet as well as or Internet users for monitoring and setting new values of temperature and humidity through the respective front end.

\section{Introduction}

Microcontroller based embedded systems designed for monitoring and controlling of parameters like temperature and humidity is very essential in some industrial as well as research setups [1,2]. In view of limitations of standalone embedded systems for controlling and operating from the place of its installation, interconnection of same with TCP/ IP networks can provide a broad interactive use for monitoring and controlling from the different client installations [3]. Basic objective of the system is to provide monitoring and controlling of temperature and humidity to Intranet and Internet users [4]. Both technologies work in a different manner. In client server technology users continuously receive the updated values of sense temperature and 
humidity as per constant request of client to server for updated values. whereas Internet users using the web technology will avail the same implemented in a three tire system of web browser, web server and embedded server. Due to stateless nature of web Internet users will not receive continuous updated data. Users of the system must send a HTTP request from web browser to web server then same will be forwarded to embedded server for retrieving the updated values. Both types of users can set the values of temperature and humidity through their respective front ends at which necessary control will be initiated by controller board through relays. Internet users opting for setting new values for controlling temperature and humidity will be authenticated after entering the password for same. Authenticated process is implemented through installing Mail server. Hardware control will be initiated when sense values of temperature and humidity will exceed the set values stored in limit value table. System can generate report and graph of recorded temperature and humidity as per request of users.

\section{Hardware configuration of Boards and Ex- perimental Setup}

Basic hardware design consist of two circuits monitoring board and controlling board are interconnected with Embedded server through two COM ports COM1 and COM2. Both the boards are based on Atmel 89S52 microcontroller. ADC0809 is used in monitoring board for conversion of analog transducer values to digital value. Transducer here stands for temperature and humidity sensor. Diagram along with its nomenclature of different items as given in the Figure2.1 will explain the intended purpose of same.

Monitoring board will continuously sense the parameters(Temperature, Humidity) and make it available at COM1 port. Program designed in Visual Basic will read the comport and store the sense values in the Database server designed in Mysql. Clients on the same LAN can continuously display the sense values . This is implemented by sending request to embedded server at a regular interval for updated values based on client server technology. Necessary front end designed in clients of LAN will enable users for continious monitoring of same. System will continiously check the sense values against some stored limit values of parameter at which necessary control will be initiated. Limit value table is used to store these control values which may be modified by user through interactive front as required. As soon as the sense value will cross the limit value control signal will be passed through the COM2 port to which controlling board is connected. Controlling board is responsible for carrying out necessary control by switching on/off some hardware device(such as coolers, dryers ) which are connected through relays of controlling board.

\section{Monitoring Circuit Board}

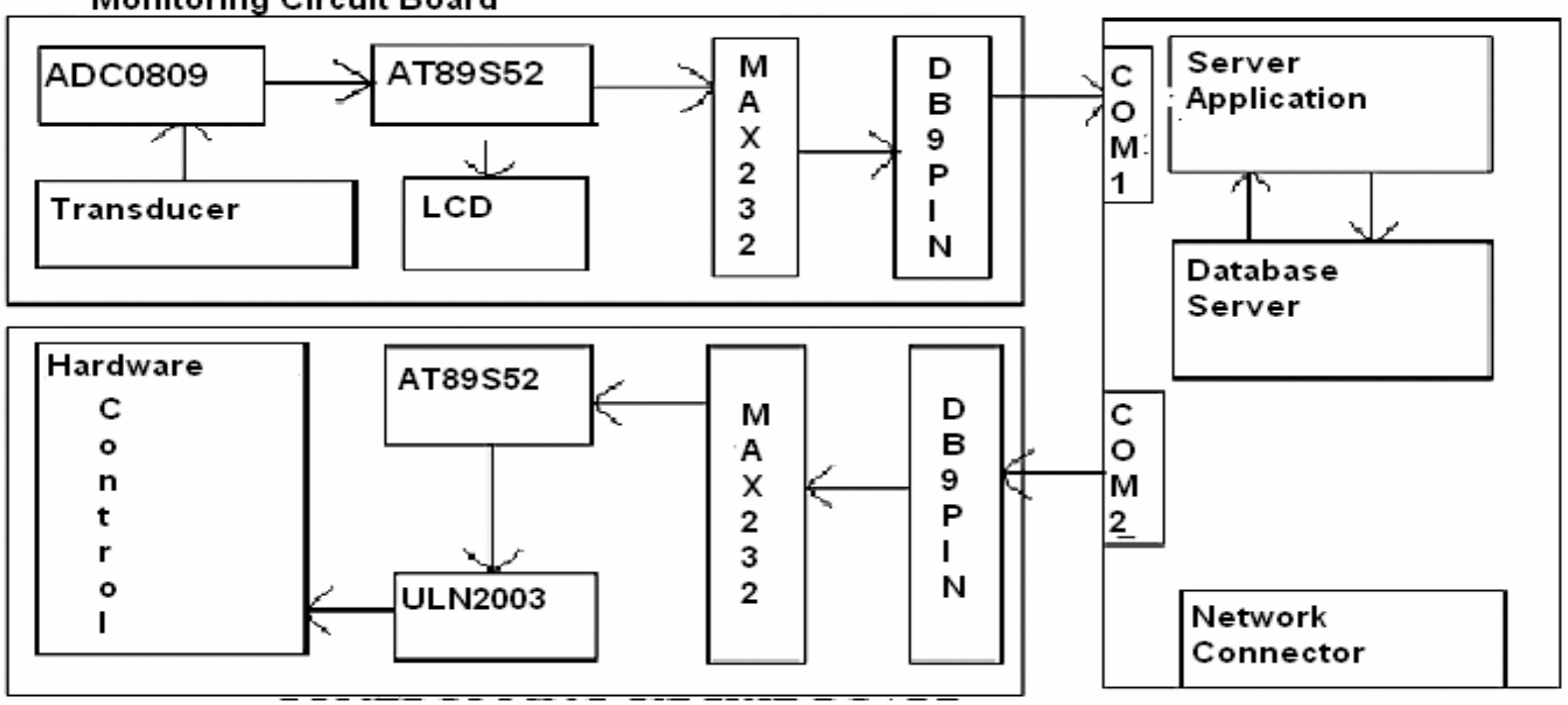

Controlling Č írcuit Board

EMBEDDEDSERVER

(Computer with database + Application to send / receive data to/from database)

Figure 2.1 Hardware configuration of boards with Embedded Server 
Internet users not belonging to the LAN where embedded server is connected can access updated values of sensor through browser. Web server IIS, Internet Web Client run the client program anywhere from the web for displaying interactive front-end for monitoring and controlling through browser. The program is implemented in C\#.NET. Experimental setup used for implementation of the system developed given in Figure 2.2. LAN/Intranent indicate the Network where Embedded server is installed with monitoring and controlling boards. This Intranet is connected to Internet backbone to make it accessible from anywhere. Mailserver and Webserver is necessary for Internet users of the system.

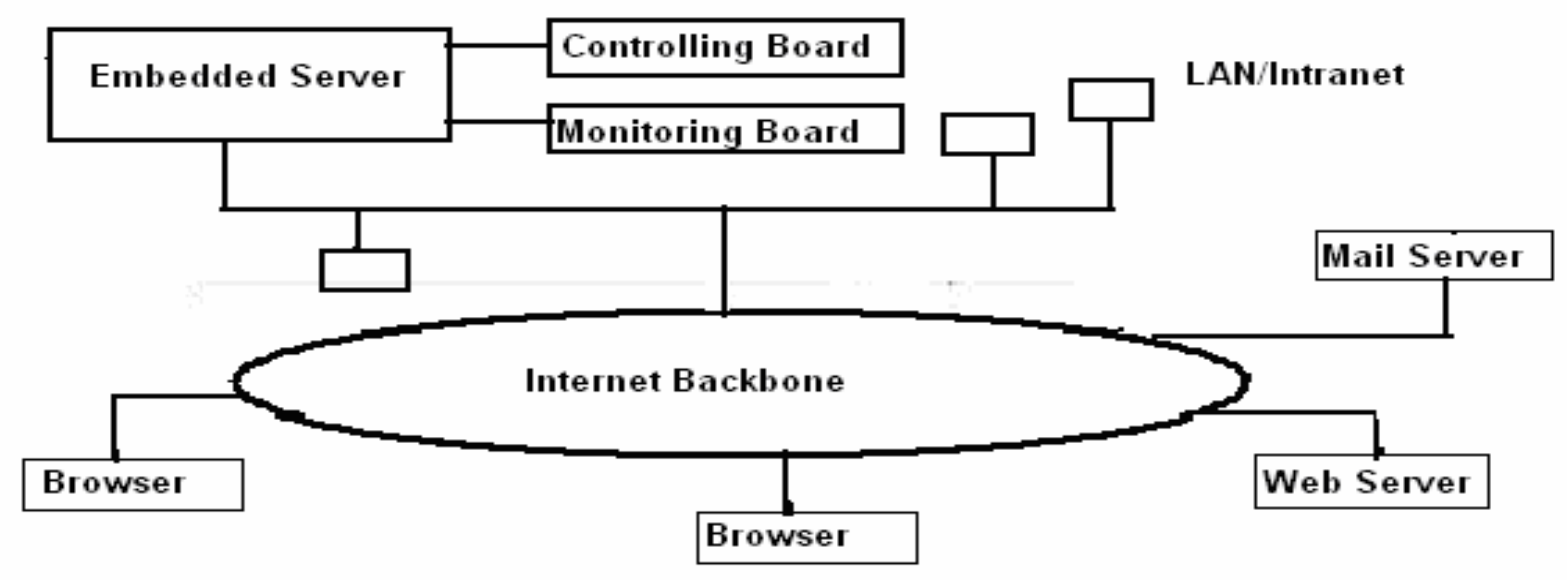

Figure 2.2 Experimental setup

\section{Schematic description of the functioning components}

a. Power supply section: The regulated power supply section made with full wave rectifier (with IN 4007 diodes) using voltage regulator IC 7805 and IC 7812 which provide a constant voltage of $5 \mathrm{~V}$ to the circuit as well as constant $12 \mathrm{~V}$ to relays.

b. Analog to digital conversion section: Analog parameters temperature and humidity are converted into digital by ADC 0809 which is a 8 channels microprocessor compatible ADC with easy availability. It will convert the analog signal of the transducer to digital value with respect to the reference voltage which is $2.5 \mathrm{~V}$ in proposed system. This reference voltage is obtained using TL431, which is a programmable shunt voltage reference with output voltage range of $2.5 \mathrm{~V}$ to $36 \mathrm{~V}$ and works like zener diode [5]. For the conversion ADC requires a reference frequency which is supplied from $555 \mathrm{IC}$ in the form of astable oscillator. The conversion frequency is kept around $150 \mathrm{kHz}$. Sensor used for temperature measure- ment is LM 35.LM 35 is calibrated in ${ }^{\circ} \mathrm{C}$ and is linear in +10 $\mathrm{mV} /{ }^{\circ} \mathrm{C}$ scale factor with $0.5^{\circ} \mathrm{C}$ accuracy [6]. It uses humidity sensor LM324DG [7]. Amplifier circuit is used to amplify the electrical characteristic obtained through the transducer to raise the strength sufficiently

c. Controller section: The analog value is converted to digital value by $\mathrm{ADC}$ and is picked up by microcontroller AT89S52 which is a 40pin device. The AT89S52 is a lowpower, high-performance CMOS 8-bit microcomputer with $8 \mathrm{~K}$ bytes of Flash programmable and erasable read only memory (EPROM) [8].

d. Display section: In addition to continuous display of sense temperature in the front end screen of computer in hardware circuit(a) on board 44780 LCD is used which is a $2 \times 16$ line display $[9,10]$.

e. Temperature control section: This section consists of a $12 \mathrm{~V}$ relay to control hardware to start cooling for maintaining temperature as set by the user. [11] 


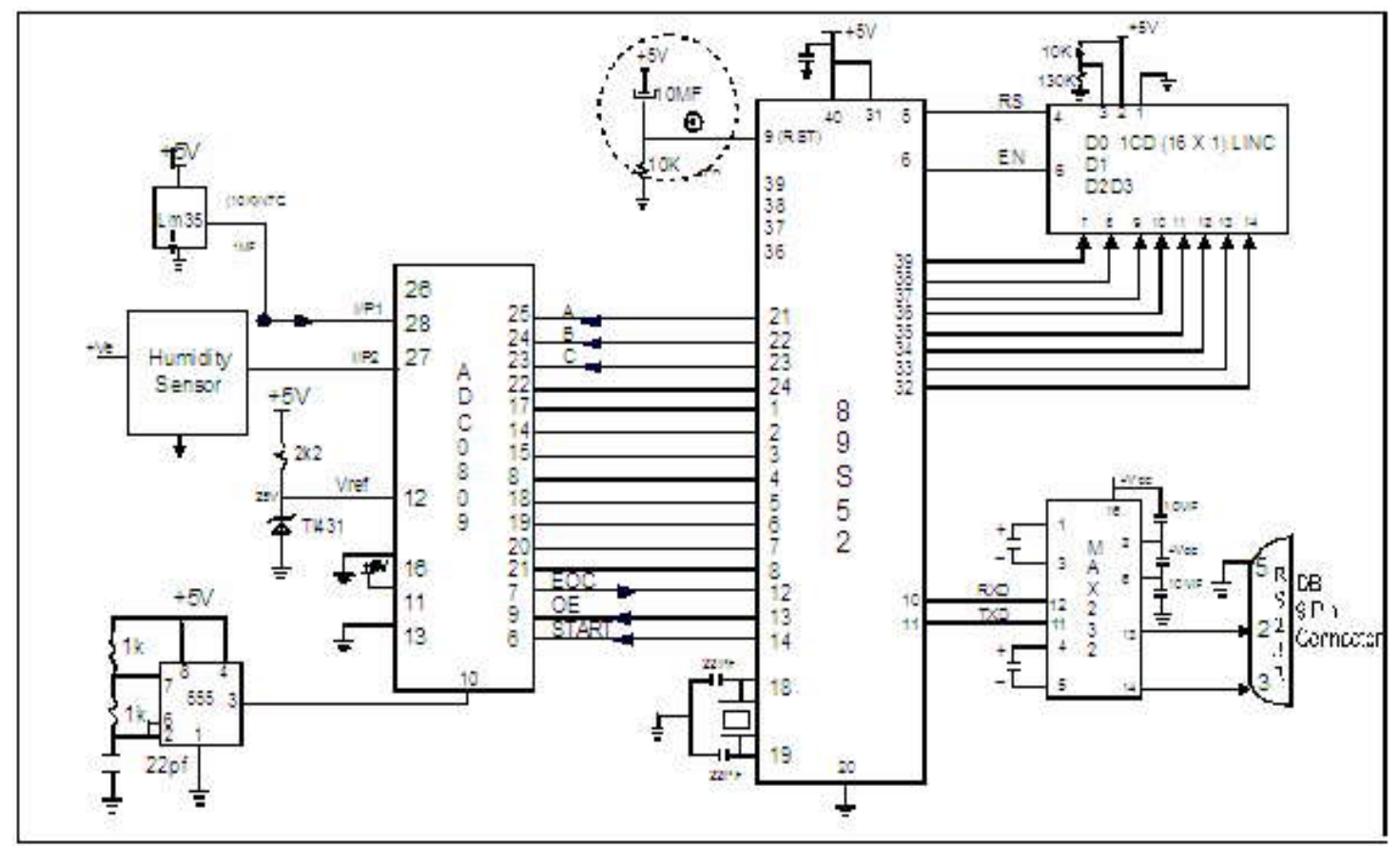

Schematic Diagram(Monitoring Circuit Board)

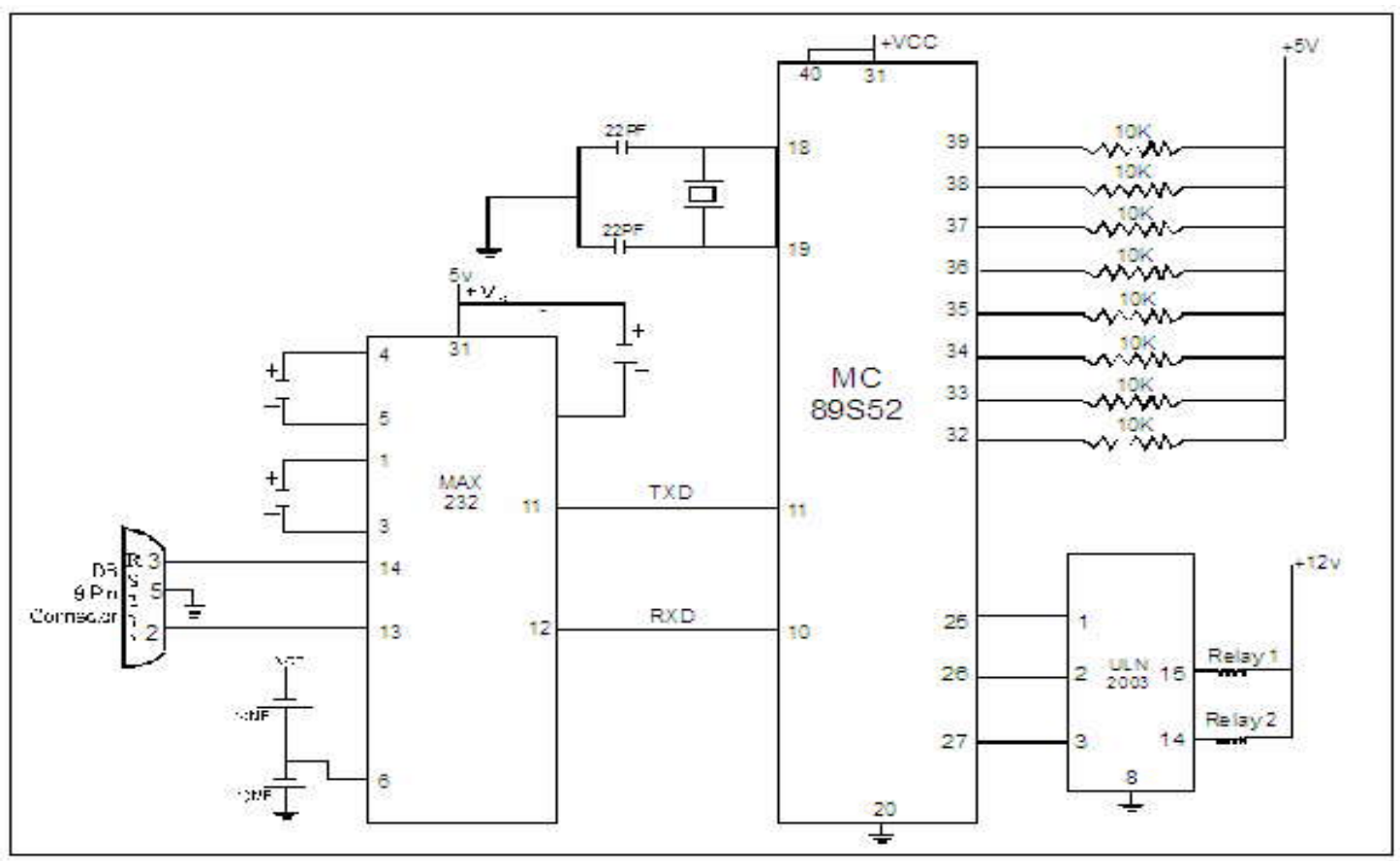

Schematic Diagram(Controlling Circuit Board)

Figure 3: Schematic Diagram 
f. Data transfer: Displayed temperature is transferred to RS 232 which is interfaced with microcontroller through MAX232 [12, 13]. In this implementation monitoring board can be considered as transmitter as after converting the sense temperature into digital form it will transmit the same to RS232 . A program executed simultaneously in computer will read and display the same in the front-end designed in the monitor. Additionally front-end will display temperature set value and status(on/off) of hardware device and enable user to enter new value of temperature in the database generate curve of temperature with respect to time. Controlling board will continuously compare sense data with set value and run hardware device for controlling temperature and humidity. Microcontroller of controller board is connected with relay through ULN2003[14] to amplify the required current necessary to drive the relay.

\section{Software Descriptions}

Four different software modules execute simultaneously for monitoring and controlling of temperature in the present work. . A brief description of functioning of each module is given below

Module1: Contains the software burnt in to the microcontroller of the monitoring board. This program read sensor data (temperature and humidity) obtained from ADC and converted into appropriate form of display in LCD. This program carryout the proper calibration before display of data. It will also transmit the same data to RS232 port attached in the circuit. Software is implemented in $\mathrm{C}$ language. This program monitor as well as read a particular sensor value for a few millisecond before switching to other sensor defined a zone. It directs the interrupt generated by microcontroller to be sent to appropriate ISR(Interrupt Service Routine), such as timer interrupt and serial interrupt. These routines are executed whenever timer0 time out and a byte is transferred to serial port COM1. Baud rate is fixed at $9600 \mathrm{bps}$. Program also responsible for initializing microcontroller registers P0,P1,P2,P3, TL1, TH1, IE0, SCON etc with required values. It calls appropriate routines for calibration and conversion of binary values generated by ADC to ASCII values and display them. It also calls delay routines to avoid overlapping such as calibration of sensor value and reading sensor value to achieve good performance

Module2: Contains the software burnt into the microcontroller of controller board. This program is responsible for running appropriate hardware devices through relay if sense temperature and humidity reaches the set value of temperature and humidity. Program signals the appropriate relays by setting flags say Flag_relay1, Flag_relay2. The ISR is invoked whenever data arrives at COM port. , it set appropriate flag after storing data in array. Microcontroller then sent control data to through its appropriate pins to relay for switching( ON/OFF) connected hardware device.

Module3: Contains the software that runs synchronously with monitoring and controlling board. It runs on Embedded server where database server is implemented in Mysql. This program is implemented in Visual Basic 6 and interact with database server. It displays continuously the sense temperature and humidity in calibrated form. This software retrieves the sense data of temperature and humidity and stores the same in Temperature and Humidity tables of the database with corresponding date and time. It also store user entered set value of temperature and humidity in a separate table based on which control action is initiated. This software enable its user to generate report on recorded temperature and humidity for a particular day as well as generate graph of same based on choice of user in the interactive front end User from the database server interact with database two ways -using the software of the module 3 or through the browser discussed in the module4.The functonal diagram of server process is given in figure 4

Module4: Contains software for providing web services. It consist of IIS web server with .NET framework and web application, database server and a browser as a client. The web application is implemented in C\#.NET. It sends requests to the database server to send updated data for sending the same to client as requested from client through its browser . Client may send HTTP request to Web server for getting updated values of temperature and Humidity or control data for change of new set values of temperature and humidity. Next Web server send request to Embedded server for retrieving updated values from database or setting new values of temperature and humidity for initiating control. Embedded server send updated values or new set values to web server. Web server in the form of HTTP response send the same to client The application program running at embedded server initiate necessary control by looking at the changed values in the database server. The user can view the maximum values of sensed data and the current set values upon request from the database. The advantage provided by such a web service is that the user can view and change the necessary values from any location. Performance of 


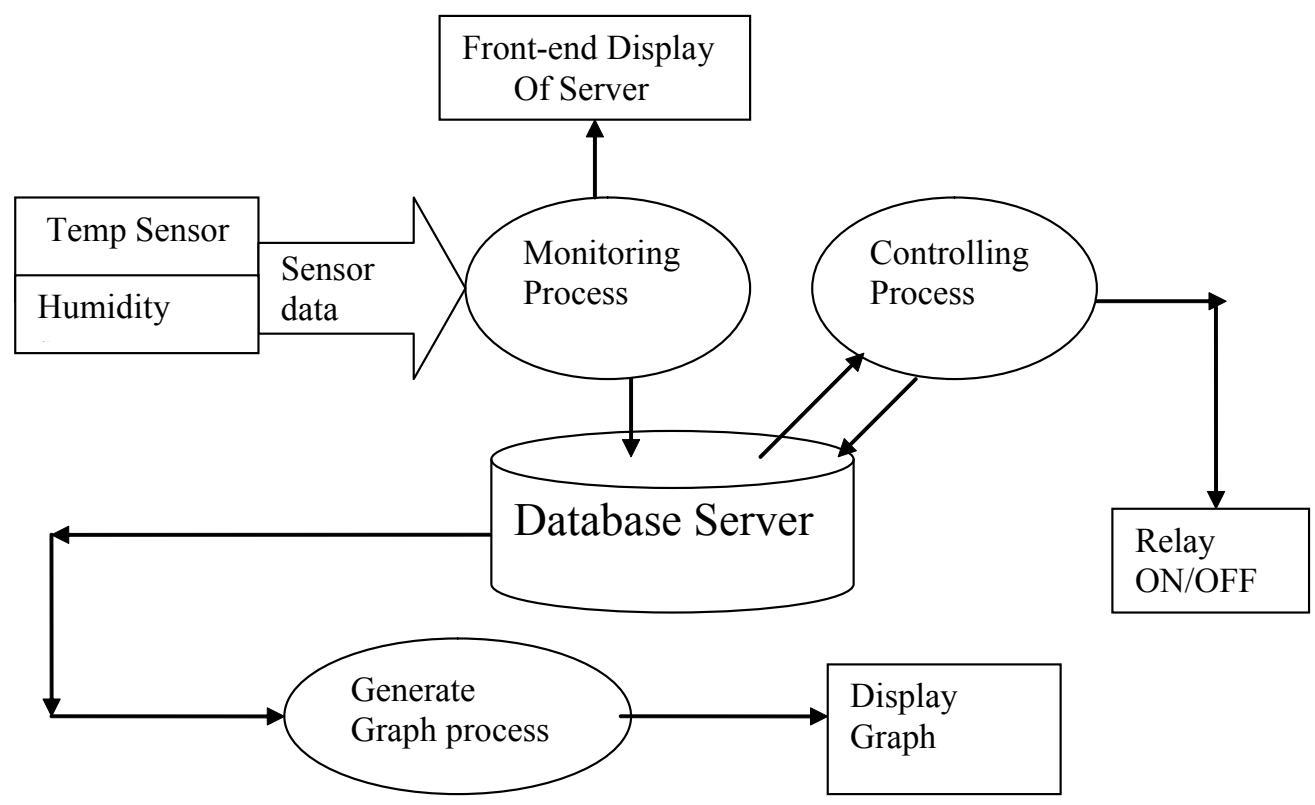

Figure 4 Functional diagram of server process

the system from the remote client depends on Network speed which is directly dependent on allotted bandwidth and congestion level of route. In case of very busy network if required bandwidth is dedicated seamless online performance will be observed..

\section{Some Issues of the System Implementing from LAN and Web}

It is already mentioned that developed system can function from both LAN and Internet/Web. As given in the figure 5.1. Some advantages as well as shortcomings has been observed during its implementation. To look into these issues it is necessary to consider client server technology and web technology which is based on TCP/ IP model. In basic TCP/IP model to establish connection TCP software on server execute a process called passive open enabling clients to establish connection on request. As clients always initiates a TCP connection by sending request to server, so client is said be active open in contrast to passive open of server. In the System implemented from LAN, as soon as client issues an active open request, local TCP software provide IP address and TCP part of the destination server application. TCP software on client uses this information to open a connection with remote server.
In LAN/Intranet implementation as client send continuous request to server at regular interval which enable its clients for monitoring of updated values of temperature and humidity in front-end of monitor. Continuous appearance of values in monitor occur as after connection establishment server gets request for updated data at regular interval from client. At the same time it also make it feasible for graph representation of temperature and humidity without any distortion. Client in LAN works as a fat client. Visual Basic implementation in client can send request at regular interval making continuous display possible. In Web implementation interactive front designed for browser send request to webserver which indeed awaits passively. User using a browser request for a webpage to web server. Web server application is built in C\#.NET will retrieve appropriate data from database. In case modification of controlling value it will modify appropriate table of database. Here request response model is governed by a protocol called HTTP in both client and server. HTTP by nature is stateless. When HTTP client prepares the request for webpage, on HTTP server, server interpret such request to pass response to client. After sending the requested page server will release the connection. Unlike VB client implemented in LAN it is a thin client governed by stateless HTTP protocol, so for updated value user have to send another request, hence the continuous appearance of values in the front-end like Intranet is not possible. 


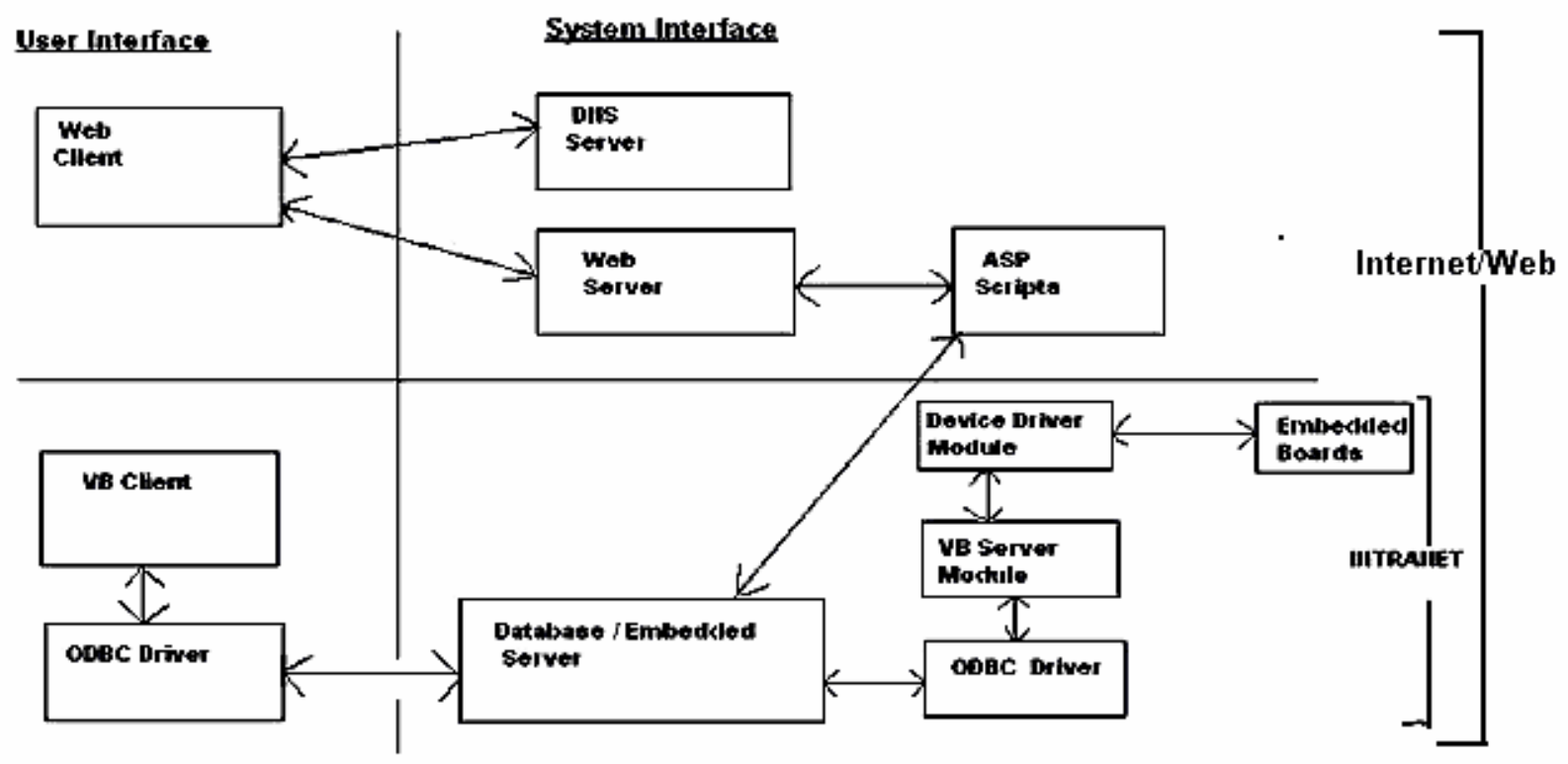

Figure 5.1. Functional diagram of the system developed

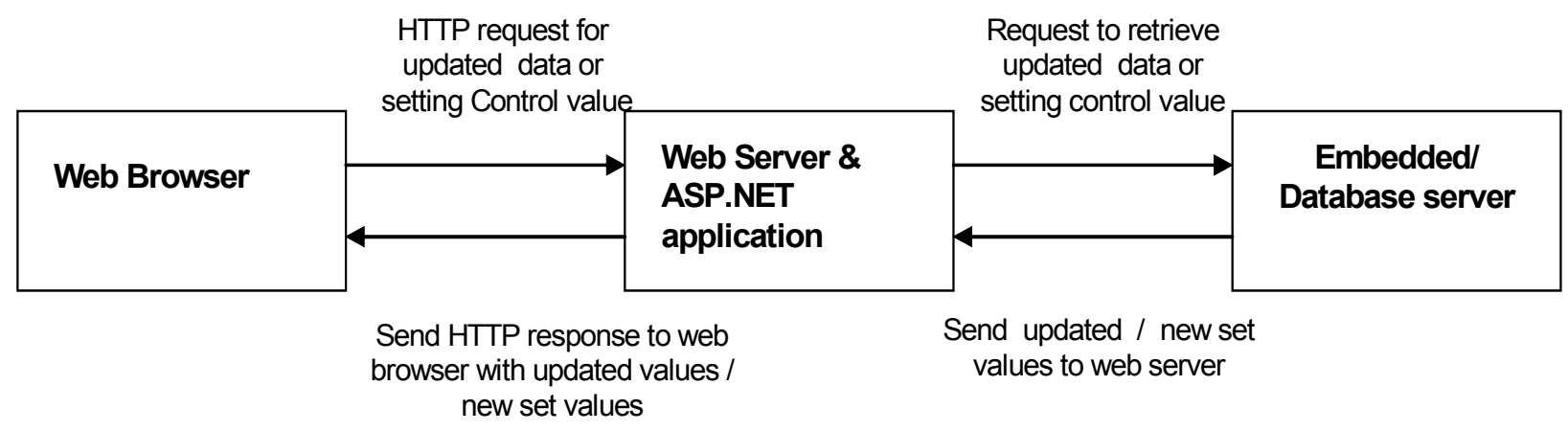

Figure 5.2. Information Exchange in the stated three tire model 
This phenomena of information exchange is shown in the three tire model of figure 5.2. Interactive front end as displayed in monitor is shown in Figure 6.1 ( for Intranet) and in Figure 6.2 ( for Internet ). One significant advantage of web based solution is that it is platform independent. An application from webserver can be viewed as web browser from any Operating System. As far as security issues are concerned, web implementation performs user authentication through a mailserver, if user wants to change the control values of humidity and temperature. In LAN implementation same can be achieved by assigning user authentication at update button of Figure 6.1.

\section{Monitoring and Controlling from Intranet and Internet}

Monitoring and controlling from Intranet(LAN) using the front end as in figure 6.1, Client being fat in nature will send continuous request in regular interval to the server for updated values of temperature and humidity . Server in response to same send the updated values in regular basis and monitor will displayed these values continuously. System will highlight parameter(Temperature/ Humidity) name in bold if it is currently displayed. Date and time will be displayed in top for recording of same.
Status of devices on/off will be displayed in bottom which will run automatically based on controlling limit values of parameter stored in database. It will show the status message regarding values of current sensing parameter such as (Humidity reaches maximum value as appearing in the screen will be displayed). As soon as parameter will come down to controlled level device will automatically come to switch off mode and message will disappear. Maximum range gives the information of the value currently set for the controlling parameters. Using update button it will enable user to change the limit values in the respective database. Clear Humidity and temperature button will clear the values of parameters from the screen. End program will halt the execution of program though in normal circumstances it will be not requiredGenerate report button enable user to generate report based on temperature and humidity of a particular day as chosen by user. These will be retrieved from the database with corresponding date and time of recorded . Selecting Temperature or humidity from the drop-down list of the generate report will display the report for the selected option

Monitoring and controlling from Internet(WEB), using the front end as in figure 6.2 enable users to interact with the embedded system from anywhere in the web .

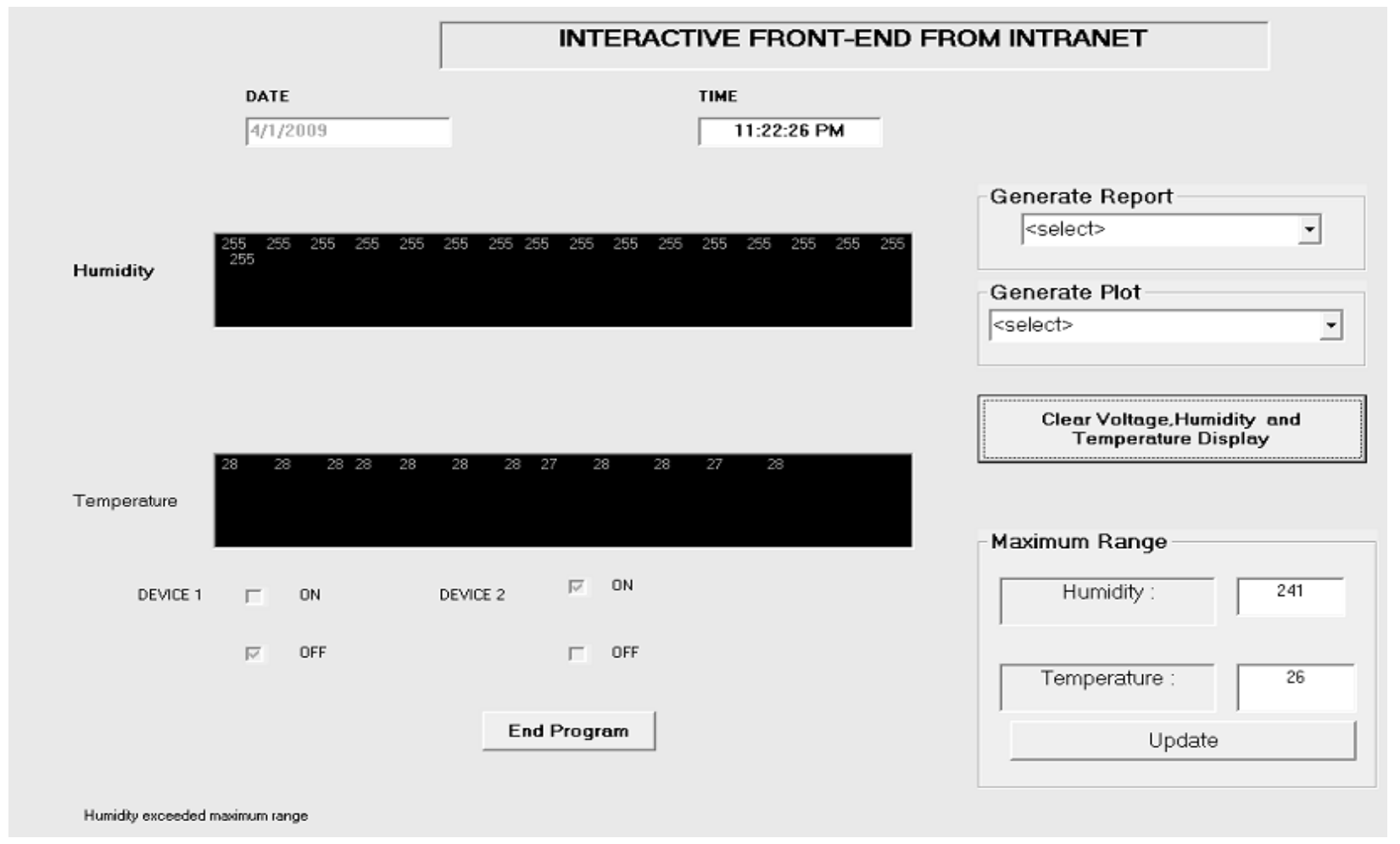

Figure 6.1 Interactive front-end from Intranet displayed in monitor 
User as a web client select a particular date from available list and click on submit button for getting maximum temperature and humidity recorded for that particular date. Refresh date will enable user to refresh date. Clicking on get current values browser will sent request to web server for getting updated data of humidity and temperature. It will display the existing limit values of temperature and humidity in the space provided along with set temperature limit and set humidity limit when user click on show limit button.

To prevent unauthorized users from unwanted updation by setting new values of temperature and humidity system checks the authentication of users. System will allow user to set new control, for which user has to login with valid user-id and password as given in login form below which will appear after clicking in login button. This is implemented by designing a mail server where database server keeps track of informations with login-id, password and email-id of authorized users in a separate table. Administrator of the system will issue login-id and password to internet users of system authorized to set new values of temperature and humidity. These authorized users can also change the existing password. If the user forgets his login-id password, he can use forgot password form for retrieving the same. User will receive the same in his registered e-mail id from administrator . Mail Transfer Agent (MTA) used for sending mail, Sendmail is used for sending message for mail server. This facility is provided in ASP.NET

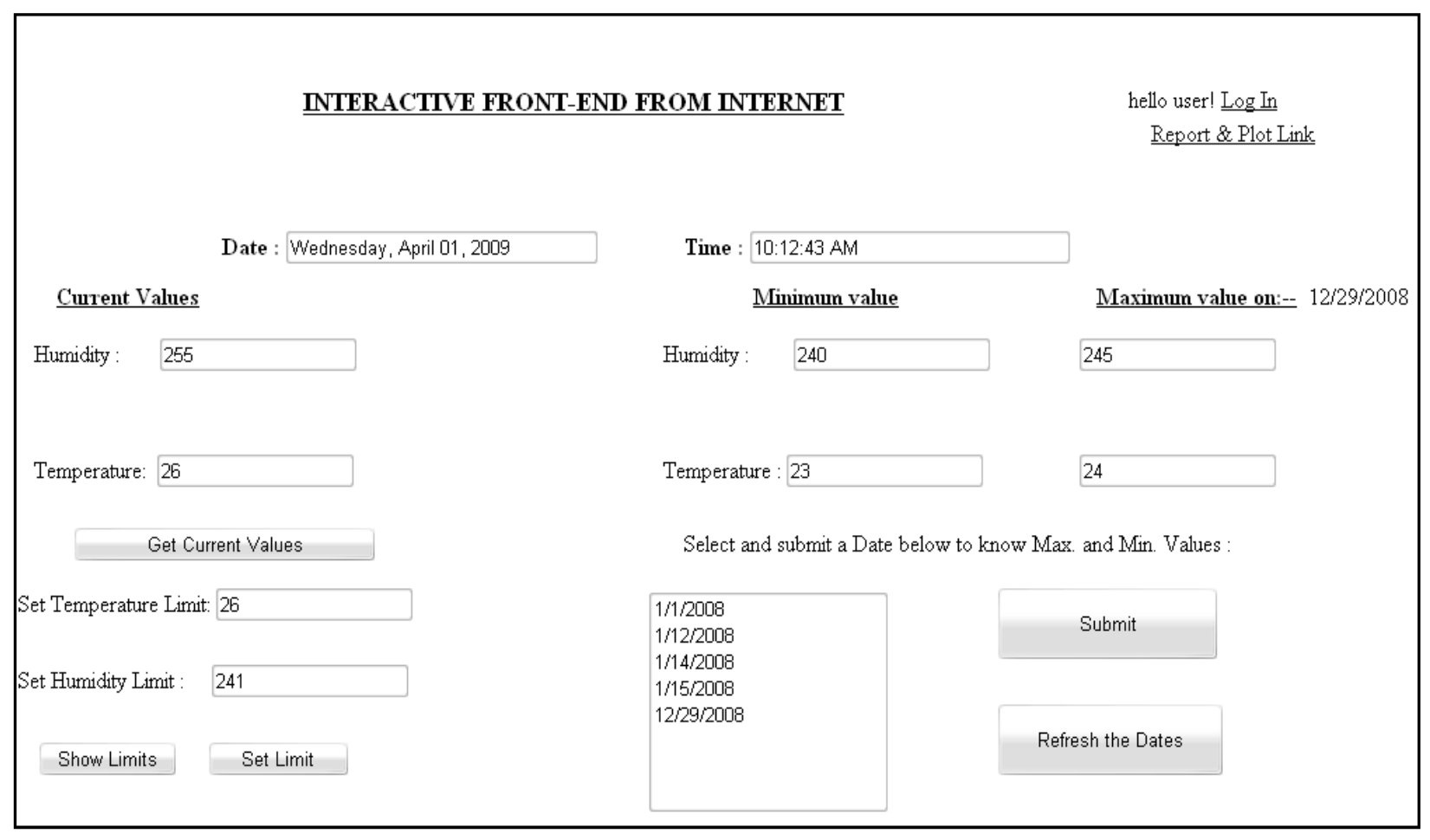

Figure 6.2 Interactive front-end from Internet displayed in monitor

\section{Performance and Conclusion}

Performance of any system depends on accuracy. Indeveloped system performance is monitored in two levels,accuracy and smooth operation. Accuracy indicate howclosely the sensor can measure the real world values.

Acceptable result is observed on monitoring. Performanceof the remote clients of Intranet and
Internet depends on Network speed which is directly dependent on bandwidth allotted and network traffic. Allotment of fixed channel for Internet user can provide a seamless online performance.Developing same using 16 bit or higher microcontroller in embedded linux plateform will enhance the performance and enable it for inclusion of features like scheduling with priority concept. 


\section{References}

[1] A Review of PC-Based Data Logging and Recording Techniques http://zone.ni.com/devzone/cda/tut/p/id/2693

[2] Muhammad Ali Mazidi and Janice Gillispe Mazidi, The 8051 microcontroller and embedded systems, Pearson education ltd.,India, 2007.

[3] S. Baruah , A. Kakoti Mahanta, K.C. Sharma, "A LAN based PC Interactive Embedded System for monitoring and controlling Temperature.", Proceedings of 2008 International Conference on Embedded Systems and Applications, WORLDCOMP'08 14th-17th July, 2008, Las Vegas Nevada, U.S.A.pp.133-139

[4] S. Baruah , A. Kakoti Mahanta, , K.C. Sharma, "Web Enable Multichannel Embedded System For Monitoring and Controlling Temperature and Humidity", International Journal of Emerging Technologies and Applications in Engineering, Technology and Sciences"(IJ-ETA-ETS), ISSN:0974-3588 Amoghsiddhi Education Society, Sangli -416 415MH, India., July 08 -December 08 pp 91-100.

[5] National semiconductor corporation, ADC 0809 data sheet, 8-bit Microprocessor compatible A/D converters with 8-channel multiplexer, national Semiconductor data book, October 2002 up-dates. http://www.national.com/mpf/DC/ADC0809.html
[6] National Semiconductor Corporation, LM35 datasheet, precision centigrade temperature sensors, Atmel data book, November 2000 update.http://www.national.com/mpf/LM/ lm35.html

[7] http://www.ti.com

[8] Atmel corporation, AT89S52 data sheet, 8-bit microcontroller with $8 \mathrm{k}$ bytes flash, Atmel Data book, 2000 update.

[9] http://en.wikipedia.org/wiki/Liquid_crystal_display .

[10] Introduction to LCD programming tutorial by Craig Steiner Copyright 1997 -2005 by Vault information services LLC (http:/ /8052.com/tutlcd.phtml)

[11] kalsi. H S , "Electronic instrumentation", Tata McGrawHill Ltd., New Delhi, 1999

[12] RS232 Interface using MAX232, written by Peter Luethi Dietikon, Switzerland, Date-23-Apr-2001, Page-7 of 8, Revision-1.03

[13] www.maxim-ic.com

[14] www.alldatasheet.com/ULN2003/TEXAS 